\title{
Superficial CD34 Positive Fibroblastic Tumour, A Mesenchymal Tumour with Borderline Malignant Potential: A New Entity
}

\author{
Gurpreet Kaur Walia*, Prateek Kinra and Ajay Malik \\ Department of Pathology, Armed Forces Medical College, Pune
}

\section{ABSTRACT}

Superficial CD34 positive fibroblastic tumour (SCD34FT) is a distinct mesenchymal tumour of superficial soft tissues which has an intermediate malignant potential and is a recently introduced entity in the WHO classification. We present a case report of a 32-year-old male patient with SCD34FT. The tumour was located on right forearm and was essentially superficial in location. Wide local excision of the tumour was done. On histopathological examination, the tumour was composed of highly pleomorphic spindled as well as epithelioid cells with granular eosinophilic cytoplasm and numerous bizarre lobulated nuclear forms with few showing intranuclear inclusions. However, the mitotic count was very low. On immunohistochemistry (IHC), tumour cells were diffusely positive for vimentin and CD34 (characteristic of SCD34FT). During clinical follow-up, the patient is asymptomatic since the time of his surgery one year back. SCD34FT is a mesenchymal tumour of borderline malignant potential which is restricted to the superficial location

Keywords: Superficial CD34 Positive Fibroblastic Tumour, Pleomorphism, Epithelioid, Myxofibrosarcoma, Immunohistochemistry.

\section{Introduction}

Superficial CD34 positive fibroblastic tumour is a recently described rare and distinct mesenchymal tumour of superficial soft tissues which has an intermediate malignant potential. The neoplastic spindle cells are arranged in fascicles and storiform pattern with tumour cells showing striking nuclear and cellular pleomorphism, but by contrast, possess low mitotic activity. ${ }^{[1,2]}$ On immunohistochemistry, this recently proposed entity shows diffuse vimentin and CD34 positivity with few cases showing focal CK expression.

\section{Case report}

32-year-old male presented with history of right forearm swelling for nine years which was insidious in onset and gradually progressive. On examination, a wellcircumscribed mobile, non-tender swelling measuring $3 \times 4 \mathrm{~cm}$ was noted which was soft to firm in consistency. There was no history of trauma. Ultrasonography of right forearm revealed a well-defined heterogeneously hyperechoic mass lesion measuring $2.5 \mathrm{x} 1.8 \mathrm{x} 3.3 \mathrm{~cm}$ in the subcutaneous plane at right forearm with internal vascularity. The tissue plane between the lesion and underlying muscle was maintained and adjacent nerve or vascular involvement was not evident.

FNAC of the lesion was advised and the smears were moderately cellular comprising of spindle pleomorphic and plump tumour cells (Fig. 1a). These tumour cells had abundant granular and vacuolated cytoplasm with nuclei showing dispersed to vesicular chromatin
(Fig. 1b). Intranuclear inclusions were also noted in few cells (Fig. 1c-d) with some cells having conspicuous nucleoli (Fig. 1d). Based on these cytology features, an opinion of a low-grade spindle cell lesion was given as the lesion had prominent pleomorphism but was not mitotically active. Excision biopsy was advised for definitive histopathological correlation and wide local excision of the tumour with sufficient margin was performed. The laboratory received a skin covered tissue bit measuring $5.5 \times 4 \times 2.2 \mathrm{~cm}$ with skin flap measuring $4.5 \times 4.5 \mathrm{~cm}$ (Fig. 2a). On cut section, a well-circumscribed and encapsulated solid-cystic lesion was noted with solid component being firm and grey white in appearance and cystic area was reddish brown in colour depicting areas of haemorrhage. No areas of necrosis were noted (Fig. 2b).

Haematoxylin and eosin-stained sections from the lesion showed a circumscribed tumour (Fig. 3a) disposed in vague fascicles and sheets (Fig. 3b). These cells were large, had spindle to epithelioid morphology with abundant granular eosinophilic cytoplasm and large hyperchromatic nuclei with smudged chromatin. These cells exhibited moderate to marked cellular as well as nuclear pleomorphism with prominent nucleoli (Fig. 3c-d). Occasional intranuclear inclusions (Fig. 3e), bizarre lobulated nuclear forms and xanthogranulomatous change was noted. The mitotic count was $1 / 10 \mathrm{hpf}$ in mitotically active areas. Stroma showed mild inflammatory infiltrate composed of lymphocytes. No areas of necrosis were seen. The base, skin margin and all four radial margins were free of tumour. 
On immunohistochemistry, the tumour cells were strongly positive for vimentin, CD34 and CD99 (focal) (Fig. 4a) and negative for bcl2, SMA, CD68, CK, MyoD1, myogenin, CD31, FLI-1 and HMB45. INI 1 was retained in tumour cells. Mib 1 labelling index was 1-2\% (Fig. 4b). Based on histopathology and IHC, a diagnosis of Superficial CD34 positive fibroblastic tumour was given with FNCLCC grading: Grade I, Stage 1A (pT1aNx).
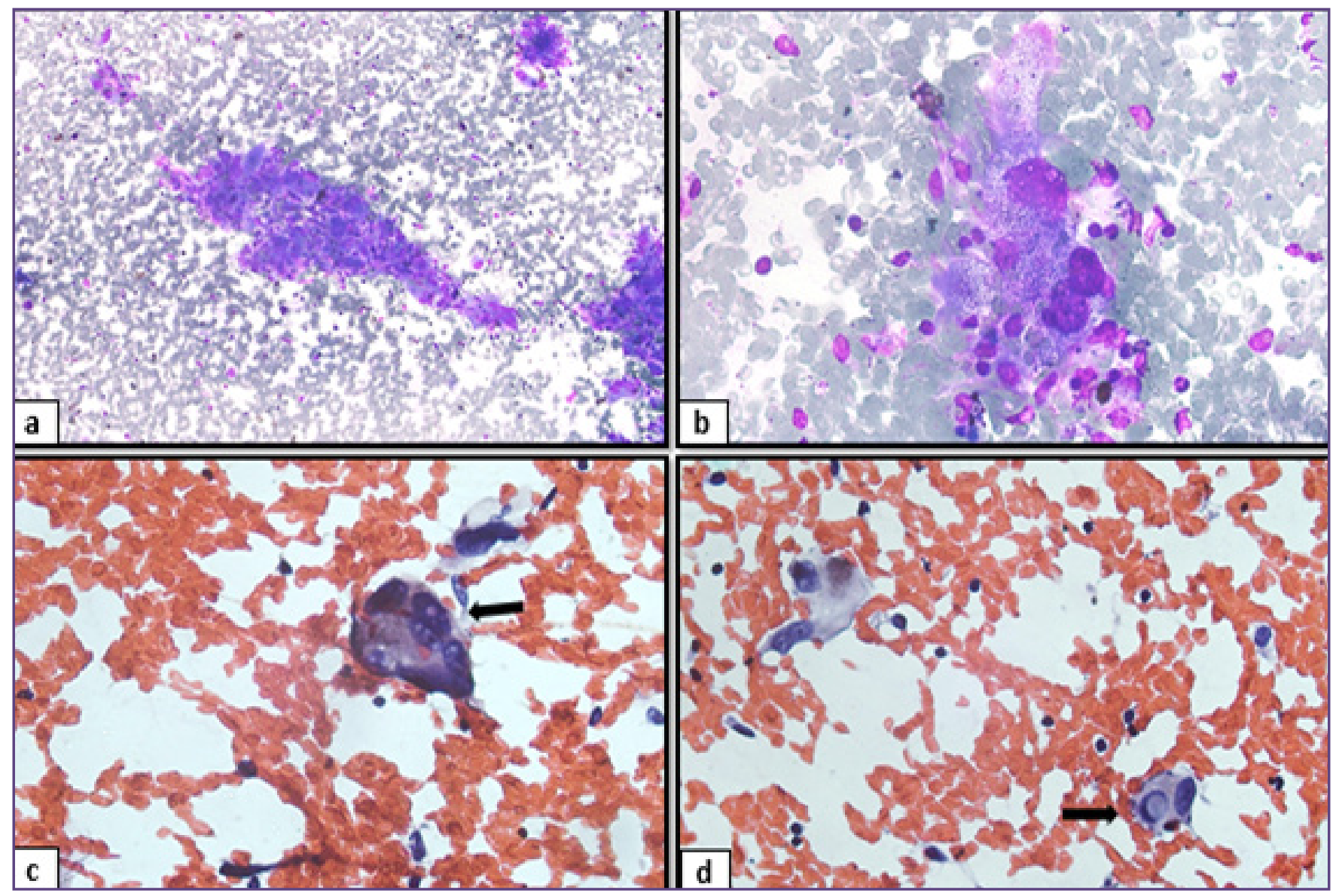

Fig. 1a-d: FNAC smears. 1a: Cellular smears showing clusters of spindle and plump cells which show pleomorphism (MGG, 200X); 1b: Cells have abundant granular and vacoulated cytoplasm (MGG, 400X); 1c-d: Large pleomorphic cells with intranuclear inclusions (arrows) and prominent nucleoli (PAP, 400X).

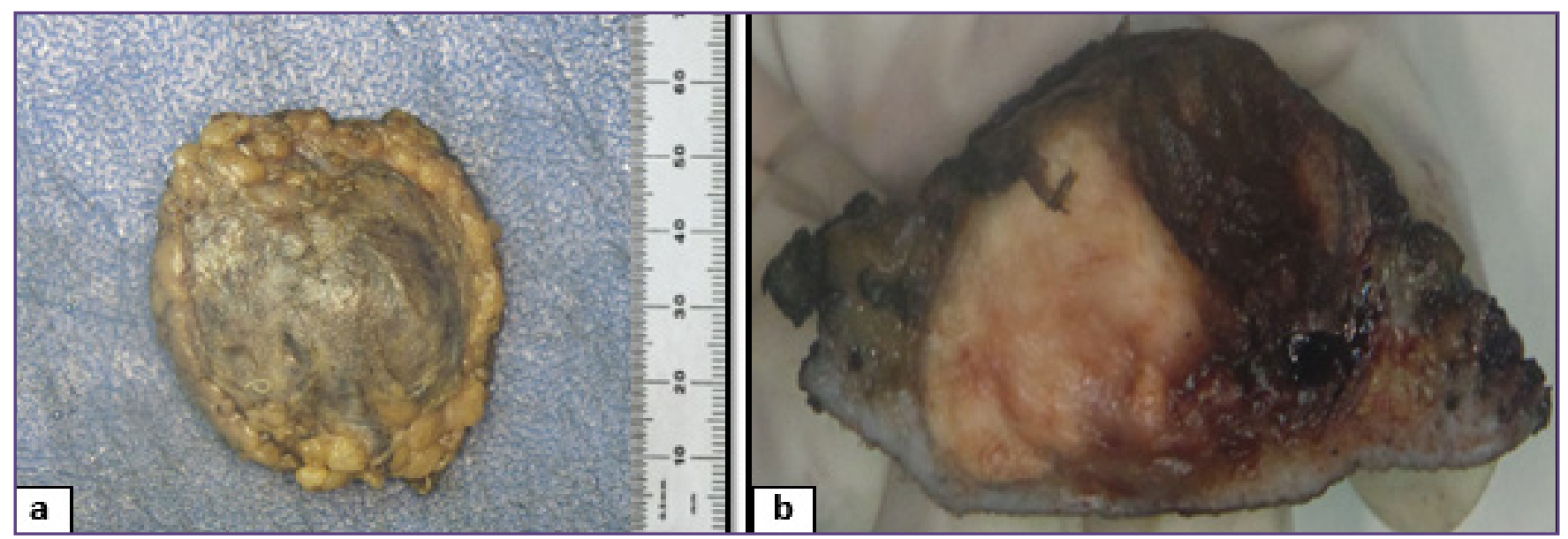

Fig. 2a-b: Gross images of the tumour: 2a: External surface shows a well circumscribed tumour; $2 \mathrm{~b}$ : Cut surface shows greyish white solid component and reddish brown cystic area with haemorrhage. 

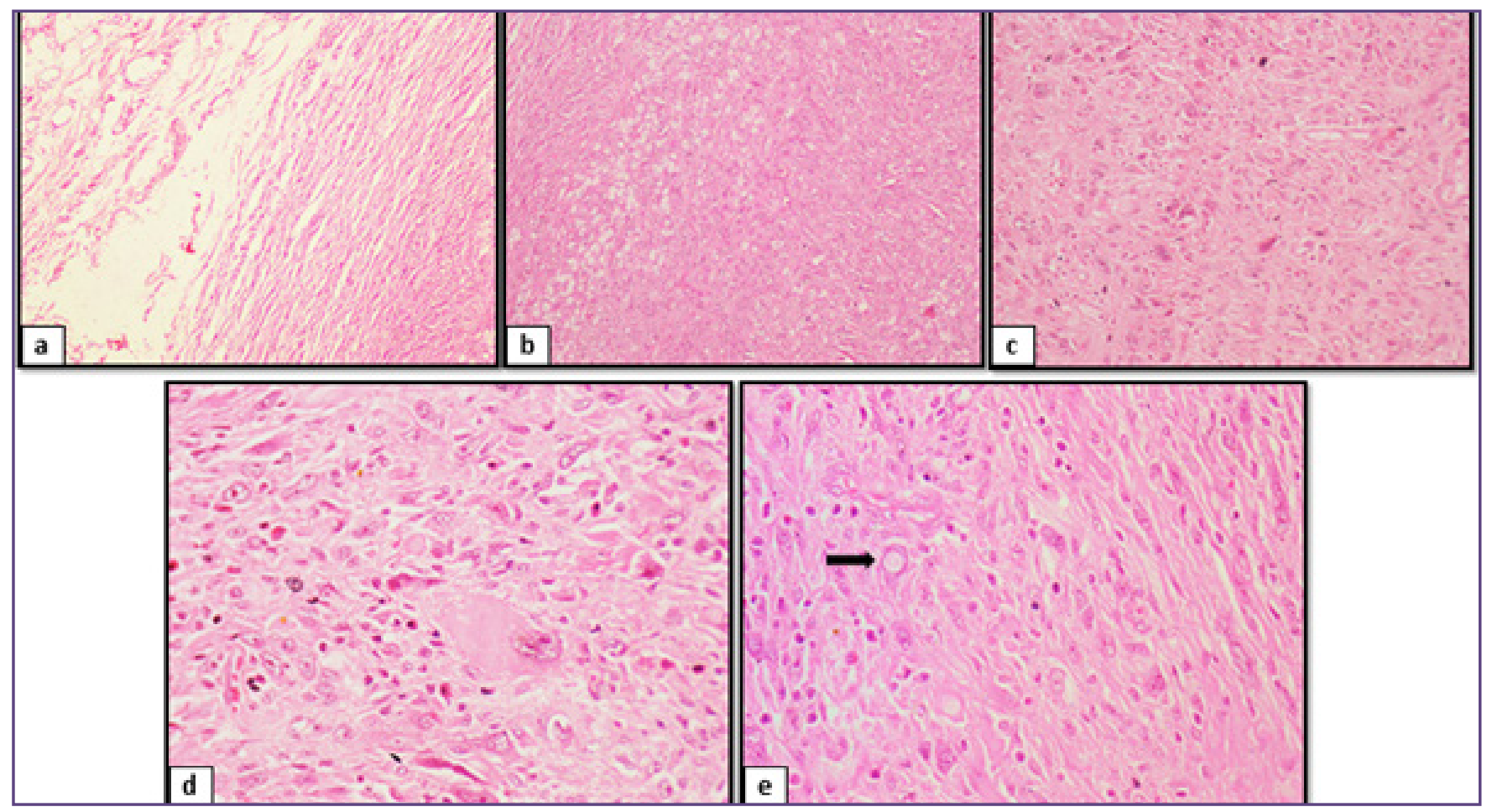

Fig. 3a-e: Hematoxylin and eosin stained sections. 3a: Well circumscribed tumour (100X); $3 \mathrm{~b}$ : Tumour cells in fascicles and sheets with xanthogranulomatous change (100X); 3c: Pleomorphic spindle to epithelioid cells (200X); 3d: Cells showing abundant granular.

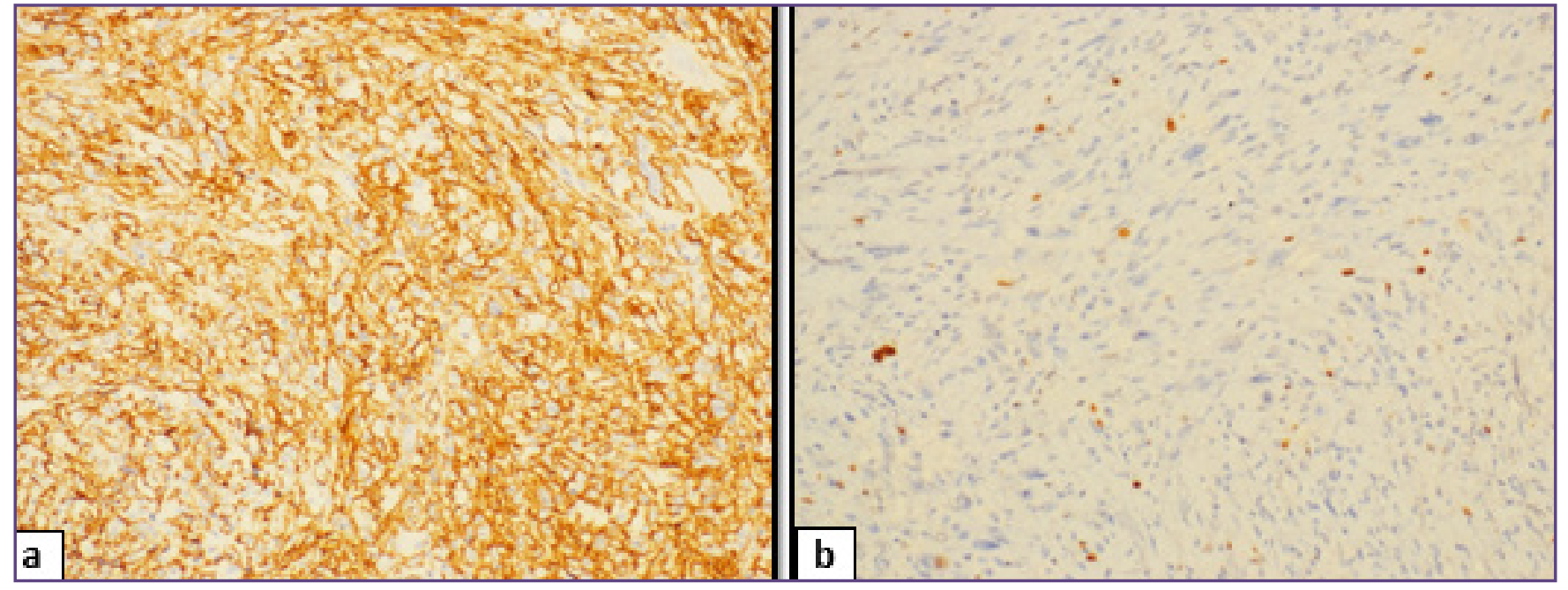

Fig. 4(a-b): Immunohistochemistry: 4a: diffuse CD34 positivity (200X); 4b: Mib-1 labelling index: 1-2\% (200X).

\section{Discussion:}

SCD34FT is a new entity introduced recently in the WHO classification which is commonly confined to deep dermis and superficial subcutis. Grossly, these tumours are relatively circumscribed but infiltrate into the adjacent tissue. ${ }^{[3]}$ This slow growing tumour occurs in middle aged adults with a mean age range of 20 to 76 years and has a slight male preponderance. ${ }^{[2-4]}$ The most common sites of predilection in descending order are: lower limbs, shoulders, buttock and upper limbs. This mesenchymal tumour of borderline malignant potential has some distinct morphological features which made it to be classified as a separate entity. This tumour shows spindled to epithelioid cell proliferation in fascicles or sheets. These cells show marked pleomorphism, and have abundant granular or glassy cytoplasm with prominent nucleoli and intranuclear 
inclusions, but still have a very low mitotic index. Also, chronic inflammatory cells along with variable amount of xanthogranulomatous change is characteristically seen. ${ }^{[3]}$ On IHC, the tumour cells are strongly positive for CD34 and vimentin, focally positive for CK and are negative for SMA, EMA, desmin, ERG, S 100, FLI -1 and melanocytic markers. Treatment by wide local excision of this mesenchymal tumour with good margins is usually curative. ${ }^{[3,5]}$ SCD34FT has a good overall prognosis but can result in local recurrence in few cases based on margin status. ${ }^{[3,5]}$ By knowing and distinguishing this new entity from various other high-grade lesions, an unnecessary radical excision and/or administration of adjuvant therapy can be avoided.

The various differential diagnoses of this tumour include- atypical fibroxanthoma, myxofibrosarcoma, undifferentiated pleomorphic sarcoma, fibrosarcoma arising in a dermatofibrosarcoma protuberance (DFSP), epithelioid sarcoma, pleomorphic hyalinising angiotectic tumour, synovial sarcoma, epithelioid leiomyosarcoma, atypical fibrous histiocytoma, solitary reticulohistiocytoma, hemosiderotic fibrolipomatous tumour and pleomorphic rhabdomyosarcoma and granular cell tumor. ${ }^{[1,2,4]}$

Atypical fibroxanthoma resembles SCD34FT morphologically but more commonly the former is located in dermal region and is mitotically more active with atypical mitosis. On IHC, atypical fibroxanthoma is positive for CD10 and negative for CD34, ${ }^{[4]}$ as opposed to our case. Genetically, atypical fibroxanthomas commonly show TP53 over-expression (not seen in SCD34FT). Myxofibrosarcoma usually occurs in older age group as a superficial tumor in extremities. On H\&E sections, this tumor shows myxoid nodules and arborizing vasculature with thick walls and lacks cellular pleomorphism. This tumor too has a higher mitotic index as compared to SCD34FT. ${ }^{[1,4,6]}$ Undifferentiated pleomorphic sarcoma (UPS) arises in deeper soft tissues of extremities of older adults and morphologically, shows admixture of pleomorphic and storiform areas with tumor giant cells. This tumor has a higher mitotic index with atypical mitotic figures and areas of necrosis. On IHC, UPS is negative for CD34, whereas our case showed strong positivity for CD34. ${ }^{[1,7]}$

DFSP is another strong differential diagnosis which has invasive and metastatic potential. Both SCD34FT and DFSP can exhibit pleomorphism with CD34-positivity in tumour cells, but DFSP shows a distinctive storiform pattern of spindle cells infiltrating into the subcutaneous tissue. ${ }^{[3,8]}$
Areas with fibrosarcomatous transformation show a typical herringbone pattern with obvious mitotic figures and reduced CD34 expression. There is significantly increased p53 and Ki-67 expression. This diagnosis was excluded on the basis of clinicoradiological inputs on superficial location of the lesion, its circumscription on gross as well as microscopy, absence of herringbone pattern (for fibrosarcomatous transformation), and low mitotic activity.

Epithelioid sarcoma can occur in superficial and deep locations in the extremities. Morphologically, irregular nodules composed of epithelioid cells are seen, that tend to merge into spindle cells. The tumor cells show minimal pleomorphism and have abundant eosinophilic cytoplasm. Stroma shows desmoplasia and areas of necrosis can be seen. On IHC, tumor cells are positive for CK, EMA, CD34 (50\% cases) with loss of INI-1 whereas it is retained in SCD34FT. ${ }^{[1,9]}$ Diagnosis of Pleomorphic Hyalinizing Angiotectic Tumor (PHAT) is based on presence of hyalinized vessels which may show thrombosis along with stromal hyalinization. ${ }^{[2,10]}$ All such features were absent in our case. Synovial sarcoma (SS) usually arises in deep soft tissue of the upper and lower limbs and is also seen in head and neck region. Histologically, the neoplastic spindle cells in monophasic SS are small monomorphic cells arranged in sheets, vague fascicles or herringbone pattern with scant cytoplasm, hyperchromatic nuclei and inconspicuous nucleoli. Also, staghorn-like vessels are commonly seen and pleomorphic cells with bizarre nuclei are uncommon in SS. ${ }^{[1]}$ On IHC, the spindle cells are strongly positive for CD99 (membranous) and TLE 1 (nuclear), focally positivity for S-100 and EMA and negative for CD34. Due to superficial location, morphology and IHC staining pattern (CD99 focal and positive for CD34), differential diagnosis of SS was ruled out.

Epithelioid leiomyosarcoma is another close differential which is predominantly composed of epithelioid tumor cells showing abundant eosinophilic cytoplasm. Also, focal spindle cell component can be seen. On IHC, tumor cells are positive for SMA and alpha-sarcomeric actin while negative for CD34, ${ }^{[1,4,12]}$ unlike our case, which was positive for CD34. Atypical fibrous histiocytoma (dermatofibroma with monster cells) is fibrous histiosarcoma with pleomorphic multinucleated giant cells, also known as monster cells. This tumor has high mitotic activity and may show atypical mitosis. On IHC, tumor cells are negative for CD34, ${ }^{[1,4,13]}$ which goes against this diagnosis in our case. Solitary reticulohistiocytoma clinically occurs as a dermal nodule with proliferation of mononuclear and multinuclear epithelioid histiocytes having glassy 
eosinophilic cytoplasm. The tumor has low mitotic index with minimal cytological atypia. On IHC, tumor cells are positive for CD68 and CD163 and negative for CD34 (as opposed to our case, where CD68 was negative and CD34 was positive). ${ }^{[1,4,14]}$ Hemosiderotic fibrolipomatous tumor is characterized by presence of pigmented macrophages and adipocytic differentiation. Both these features are absent in SCD34FT. ${ }^{[2]}$ Pleomorphic rhabdomyosarcoma involves deep soft tissue ie. skeletal muscles and morphologically, shows marked pleomorphism with multinucleated giant cells. Tumor shows low mitosis and is positive for myogenin and MyoD1 while negative for CD34. ${ }^{[1]}$ Granular cell tumor is positive for S 100 which ruled out this entity in our case.

\section{Conclusion}

SCD34FT is a recently described entity with distinct histomorphological and IHC features. Marked pleomorphism, low mitotic rate and characteristic CD34 positivity with retained INI-1 is the hallmark of this tumor which has a borderline malignant potential with low recurrence rate and good overall prognosis. Recognizing and differentiating this lesion from highly aggressive soft tissue tumors which can show pleomorphism is important to avoid overtreatment in the form of radical resection and adjuvant therapy.

\section{Abbreviations and symbols}

SCD34FT: Superficial CD34 positive Fibroblastic Tumor

WHO: World Health Organization

IHC: Immunohistochemistry

CK: Cytokeratin

FNAC: Fine Needle Aspiration Cytology

UPS: Undifferentiated Pleomorphic Sarcoma

DFSP: Dermatofibrosarcoma protuberance

PHAT: Pleomorphic Hyalinizing Angiotectic Tumor

SS: Synovial sarcoma

\section{Acknowledgements}

Nil

Funding

Nil

\section{Competing Interests}

There are no competing interests.

\section{Statement of informed consent}

The authors certify that they have obtained all appropriate patient consent forms. In the form the patient(s) has/have given his/her/their consent for his/her/their images and other clinical information to be reported in the journal. The patient(s) understand that their names and initials will not be published and due efforts will be made to conceal his/ her/their identity, but anonymity cannot be guaranteed.

\section{References}

1. Sood N, Khandelia BK. Superficial CD34-positive fibroblastic tumor: A new entity; case report and review of literature. Indian J Pathol Microbiol 2017;60:377-80.

2. Rekhi B, Banerjee D, Gala K, Gulia A. Superficial CD34positive fibroblastic tumor in the forearm of a middle-aged patient: A newly described, rare soft-tissue tumor. Indian J Pathol Microbiol 2018;61:421-4.

3. Foot $\mathrm{O}$, Hallin M, Bagué S, Jones RL, Thway K. Superficial CD34-Positive Fibroblastic Tumor. Int J Surg Pathol. 2020 Dec;28(8):879-881. doi: 10.1177/1066896920938133. Epub 2020 Jul 1. PMID: 32608310.

4. Carter JM, Weiss SW, Linos K, DiCaudo DJ, Folpe AL. Superficial CD34-positive fibroblastic tumor: Report of 18 cases of a distinctive low-grade mesenchymal neoplasm of intermediate (borderline) malignancy. Mod Pathol 2014;27:294-302.

5. Mao X, Sun YY, Deng ML, Ma T, Yu L. Superficial CD34positive fibroblastic tumor: report of two cases and review of literature. Int J Clin Exp Pathol. 2020 Jan 1;13(1):38-43. PMID: 32055270; PMCID: PMC7013377.

6. Hendry SA, Wong DD, Papadimitriou J, Robbins P, Wood BA. Superficial CD34-positive fibroblastic tumour: Report of two new cases. Pathology 2015;47:479-82.

7. Goldblum JR. An approach to pleomorphic sarcomas: Can we subclassify, and does it matter? Mod Pathol 2014;27 Suppl 1:S39-46.

8. Batur S, Ozcan K, Ozcan G, Tosun I, Comunoglu N. Superficial CD34 positive fibroblastic tumor: report of three cases and review of the literature. Int J Dermatol. 2019 Apr;58(4):416-422. doi: 10.1111/ijd.14357. Epub 2018 Dec 19. PMID: 30569527.

9. Armah HB, Parwani AV. Epithelioid sarcoma. Arch Pathol Lab Med 2009;133:814-9.

10. Carter JM, Sukov WR, Montgomery E, Goldblum JR, Billings SD, Fritchie KJ et al. TGFBR3 and MGEA5 rearrangements in pleomorphic hyalinizing angiectatic tumors and the spectrum of related neoplasms. Am J Surg Pathol 2014; 38: 1182-1192.

11. AJH Suurmeijer, D de Brujin, A Geurts van Kessel, MM Miettinen. Synovial sarcoma. In Christopher DM Fletcher, editor. WHO Classifocation of tumours of soft tissue and bone, 4th ed. Lyon: International agency for Research on Cancer; 2013. p. 213-15.

12. Yamamoto T, Minami R, Ohbayashi C, Inaba M. Epithelioid leiomyosarcoma of the external deep soft tissue. Arch Pathol Lab Med 2002;126:468-70. 
13. Luzar B, Calonje E. Cutaneous fibrohistiocytic tumours-an update. Histopathology 2010;56:148-65.

14. PR, Lee RA. Adult-onset reticulohistiocytoma presenting as a solitary asymptomatic red knee nodule: Report and review of clinical presentations and immunohistochemistry staining features of reticulohistiocytosis. Dermatol Online J 2014;20. pii: Doj_21725.

*Corresponding author:

Dr. Gurpreet Kaur Walia; Dept. of Pathology, Armed Forces Medical College, Pune;

Phone: +91 8360421494

Email: akalgurpreet@gmail.com

Date of Submission $\quad: \quad 17 / 03 / 2021$

Date of Final Revision : $08 / 05 / 2021$

Date of Acceptance $\quad$ : $\quad 20 / 05 / 2021$

Financial or other Competing Interests: None.
Date of Publication : $\quad 30 / 05 / 2021$ 